\title{
Review of Histology Results of Hand Masses: A South African
}

\section{Audit}

Christian Vyamungu ${ }^{1}$, Pascaline Fru ${ }^{2}$, Tatolo Sefeane ${ }^{3}$, Cynthia Sathekga ${ }^{3}$, Elias Ndobe ${ }^{1}$.

${ }^{1}$ Department of Plastic and Reconstructive Surgery, School of Clinical Medicine, Faculty of Health

Sciences, University of the Witwatersrand, Johannesburg, Gauteng, South Africa

${ }^{2}$ Department of Surgery, School of Clinical Medicine, Faculty of Health Sciences, University of the

Witwatersrand,7 York Road, Parktown, Johannesburg, South Africa

${ }^{3}$ Department of Orthopedic Surgery, School of Clinical Medicine, Faculty of Health Sciences, University of the Witwatersrand, Johannesburg South Africa.

Correspondence to: Dr. Christian Vyamungu; Email: chvyamungu@gmail.com

Received: 12 Nov 2020; Revised: 10 Jan 2021; Accepted: 3 Feb 2021; Available online: 9 Mar 2021

\begin{abstract}
Background: Patients with hand masses present for consultation either for pain, loss of function, or cosmetic embarrassment caused by the mass. The majority of hand masses are benign soft tissue tumors. The aim was to review the histology results of hand masses operated on at the Chris Hani Baragwanath Academic Hospital Hand Unit in Johannesburg, South Africa, to explore the relationship of the types of masses according to age, sex, side, and compare the findings with what is in the current literature. Methods: Patients operated on in the hand unit, for hand masses between April 2016 and April 2019 with histology results were included in the study for statistical analysis. Results: There were 64 males and 105 females with a mean age of $41.03 \pm 18.81$ years. The most frequent masses were ganglion cysts. Females appeared to be more affected than males by the different hand masses, but there were no statistically significant
\end{abstract}

differences. Of the 21 giant cell tumors, 15 occurred on the right hand ( $p$-value $=0.021$ ). Conclusion: The profile of hand masses at a high-volume hand unit in Johannesburg, were comparable to the reported literature. There were no significant differences between sex and diagnosis, however, there was a relationship between diagnosis and side for giant cell tumors of tendon sheaths, requiring further exploration.

Keywords: Hand masses, hand tumor, hand growth, ganglion cyst, giant cell tumor

Ann Afr Surg. 2021; 18(3): 163-169

DOI: http://dx.doi.org/10.4314/aas.v18i3.7

Conflicts of Interest: None

Funding: None

(C) 2021 Author. This work is licensed under the Creative Commons Attribution 4.0 International License.

\section{Introduction}

Hand masses account for some of the conditions presenting to a healthcare practitioner working in a hand unit (1). Most patients who present do so either because of pain and limited hand function caused by the mass or due to aesthetic concerns.

The majority of hand masses are benign, and ganglion cysts (GCs) are the most common, followed by the giant cell tumors of tendon sheaths (GCTs), and lipomas (13 ). The hand masses may compress the surrounding tissues such as nerves or blood vessels and this can result in carpal tunnel syndrome or Guyon's canal syndrome due to the compression of the median or ulnar nerve, respectively $(4,5)$. Even though malignant hand masses 
are rare, they can be devastating and generally result in amputation of a digit or the whole hand involved $(1,2)$. Hand tumors and tumor-like conditions can arise from the soft tissues or from the bones $(1,6)$. The soft tissues involved can be skin and adnexa, fat, nerves, blood vessels, and tendon sheaths (6). Cavit et al. (1) reviewed 402 cases of hand tumors and found that $89.8 \%$ of cases were soft tissue tumors while $10.2 \%$ were bone tumors. There was a female preponderance with an average age of 41.9 years. However, most studies have reported on the frequency of the different masses with only a few reporting on the statistical difference with regard to sex or side of the hand. With regard to hand masses of bone origin, enchondromas are the most frequent masses whilst malignant bone lesions are very rare (2). Besides clinical assessment, special investigations include Xrays, ultrasound, computed tomography, and magnetic resonance imaging scans (6). Treatment of hand masses ranges from observation to surgical excision, the latter being the definitive treatment form (2). There was a need to look at the local profile of hand masses at a highvolume hand unit in Johannesburg, South Africa, and to compare the findings with that of existing literature. This review of records will serve as an additional tool for the teaching of hand tumor management, as well as serving as a reference to advocate for resource allocation to the hand unit as a teaching institution.

The study aimed to review the histology results of hand masses that were operated on at the Chris Hani Baragwanath Academic Hospital Hand Unit theatre over a 37-month period from April 2016-April 2019, and to review the types of hand masses according to age, sex, and side of the hand, and to compare the findings with what is available in the current literature.

\section{Methods}

Permission to conduct this study was granted by both the Chris Hani Baragwanath Hospital Ethics Committee and the Wits Human Research Ethics Committee with clearance certificate number M191049.This is a retrospective quantitative audit that analyzed the histology results of patients operated on for masses of the hand at the Hand Unit. All patients operated on from April 2016 to April 2019 and the histology results were included in this study. To obtain data as complete as possible, the Hand Unit theatre register and the registrar theatre database were used to acquire the histology results, age, sex of the patients, and information on the side of the hand that the mass occurred on. All cases were operated on under anesthesia except for an 8month-old patient. The specimens were placed in $10 \%$ formalin solution for histopathological processing and diagnosis. Paraffin-embedding of the tissue occurred and 3- $\mu \mathrm{m}$ tissue sections were prepared for hematoxylin and eosin (H\&E) staining and immunohistochemistry. Diagnoses were based on the World Health Organization (WHO) classification of tumors of soft tissues and bone (7). Histopathological reporting of the malignant cases was aligned with international cancer protocol templates. The hand was defined as the part of the upper limb from the wrist down to the tip of the digits, the dorsum as the extensor surface, and the volar area as the flexor surface.

All data was entered into an Excel software (Microsoft Corporation, Redmond, WA, USA) spreadsheet and exported to Statistica software (Statsoft Inc, Dell, Tulsa, OK, USA) for statistical analysis. The mean \pm standard deviation (SD) and percentages for the quantitative and categorical data were reported, respectively. The chisquare $(\chi 2)$ test was used to test the relationship between the variables. A p-value less than 0.05 was considered significant. Dependent variables were the histologic types of masses, and the independent variables included age, sex, and side.

\section{Results}

A total of 169 histology results of hand masses from 169 patients were included in this study. Of all the patients, $105(62.13 \%)$ were females and $64(37.87 \%)$ were males. The mean age of patients was $41.03 \pm 18.81$ years, the median age was 40 years, and the age range was 8 months to 88 years. The mean age of female patients was $41.62 \pm 18.28$ years with a median of 40 years, and the mean age of male patients was $40.04 \pm$ 19.76 years with a median of 39 years. Of the 169 cases, $80(47.34 \%)$ were on the right side and $89(52.66 \%)$ on the left side. Soft tissue hand masses accounted for 163 $(96.45 \%)$ of cases, whilst six $(3.55 \%)$ cases were of bone 
Review Of Histology Results Of Hand Masses

origin. Of the total number of cases, $162(95.86 \%)$ of the hand masses were benign and seven $(4.14 \%)$ were malignant.

The most frequent hand tumors amounted to 39 (23.08\%) GCs, followed by 21 (12.43\%) cases of GCTs, $21(12.43 \%)$ pyogenic granulomas, $15(8.87 \%)$ cases of lipoma, 13 (7.69\%) cases of synovitis, and $12(7.10 \%)$ cases of hemangioma. The remaining cases consisted of a mix of soft and bone tissue tumors and amounted to 48 $(28.40 \%)$ cases.

With regard to soft tissue tumors, $13 / 39(33.3 \%)$ of the GCs cases were males and 17/39 (43.6\%) cases were on the right side, whilst 22/39 (56.4\%) were on the left side. Thirty-two (82.05\%) of the GCs cases were on the wrist comprising 15/32 dorsal wrist ganglions (DWGs), and17 volar wrist ganglions (VWGs). The mean age of patients with GCs was $39.18 \pm 19.45$ years with a median of 35 years. Fifteen GCTs involved the right side and six cases were on the left side, and the sex distribution was six males and 15 females. Most of the digits involved with the GCTs entailed the thumb and index finger with six and five cases, respectively. The mean age of patients was $39.52 \pm 15.99$ years with a median of 38 years. For the 15 cases of lipoma, six were on the right hand and nine were on the left affecting 12 females and three males in total. The mean age of patients was $52.8 \pm 15.51$ years with a median of 56 years for these patients. The 21 granuloma cases were found in eight males and 13 females, 11 of which were on the right whilst 10 cases were on the left. The mean age of patients was $32.19 \pm 19.30$ years with a median of 29 years.

With regard to malignant soft tissue tumors, there were three cases $(1.69 \%)$ of soft tissue sarcomas, consisting of one synovial sarcoma, one Kaposi's sarcoma, and one fibroblastic sarcoma. These were all on the left hand and affected only males. The rest of the malignant soft tissue masses consisted of two cases of squamous cell carcinoma in a male and one porocarcinoma involving a female patient. The mean age of patients with malignant soft tissue masses was $44.16 \pm 15.68$ years with a median of 43.5 years.

Nine of the hand tumor-like conditions were synovitis of inflammatory origin whilst four cases were due to tuberculosis with a positive acid-fast bacilli test. The mean age of patients was $43.15 \pm 17.23$ years, and the median was 40 years. The tumors of bone origin comprised two enchondromas, two osteochondromas, one case of myelofibrosis, and only one case of an Ewing sarcoma (EWS). The mean age of patients was $46.33 \pm 21.51$ years with a median of 49 years.

The statistical analysis of the relationship between sex and diagnosis revealed that only the GCT was significantly related with side $(\mathrm{p}$-value $=0.021)$, with $71.4 \%$ of cases diagnosed on the right side of the hand. The rest of the diagnosis results did not show any significant relationship with side ( $\mathrm{p}$-value $>0.05)$. A $\chi 2$ test of association was also conducted to assess whether the diagnosis differed by sex. There was no significant relationship between diagnosis and sex as all the diagnoses had a p-value greater than 0.05 when cross tabulated with the patient's sex. Among the 32 wrist ganglions there was no statistical difference with regard to their location $(p$-value $=0.7237)$.

\section{Discussion}

Our study found that the majority of hand masses were from soft tissues, and very few cases were malignant. As in other studies, hand masses predominantly comprised GCs, GCTs, granulomas hemangiomas, and lipomas (6). In the study by Cavit et al. (1), 402 cases of hand masses were reported, $97.5 \%$ of which were benign lesions, and $2.5 \%$ were malignant. Their study showed a female predominance with a mean age of 41.9 years. The authors also found that GCs were the predominant masses and that these were mostly found on the wrist (1). However, they did not test the sex difference statistically. Our study revealed similar trends with most of the hand masses being benign whilst very few cases were malignant. The mean age in our study was similar to what has already been published and confirmed that most hand masses presented during the second to fourth decade of life; however, the difference in sex presentation was not statistically significant. In keeping with the literature, the GCs were the most frequent hand masses and were located mainly on the wrist, although any joint can be involved $(6,8)$. No association between the diagnosis and sex or between diagnosis and sides of 
VYAMUNGU ET AL.

the hand involved was found. In contrast to the findings by Kuliński et al. (9), where there was a predominance of DWG compared to VWG, we did not find a statistically significant difference between the two.

Our study findings confirm the fact that the GCs generally occur between the second and fourth decade of life (1). Kuliński et al. (9), in their retrospective statistical analysis of 520 cases of GCs of the hand and wrist, found a female to male ratio of $2.8: 1$, but there was no statistically significant difference between females and males in terms of occurrence of the GCs in particular locations. Similarly, we found no statistical difference between the sexes.

GCT was our second most common type of tumor and this presents as a soft tissue mass that develops over a long period, sometimes up to many years $(10,11)$. Even though it is a benign lesion with only a few malignant lesions reported, it is however, known for its local destructive ability (2) (see Figure 1A, B). In general, the GCTs are characterized by an increased recurrence rate (7-29\%) (2). As in previous studies, our study showed the GCT as the second most common type of hand mass, with a female preponderance but with no statistically significant difference between the sexes. The thumb and index fingers were the most commonly involved sites. Darwish et al. (12) found that the thumb was the most affected digit followed by the index finger, however, there was no explanation for this location. Similar to our study, the female-to-male ratio was 2:1 (12). Al-Qattan (10) however, found that the index finger and middle fingers were the most involved in GCTs in a Saudi Arabian study. We found that there was a statistically significant difference between the sides, with more lesions found on the right side, a finding also reported by Tang et al. (13) in a study in Singapore. They found that $9 / 11(81 \%)$ cases of GCTs occurred on the right side of the hand, although this was not statistically tested. Furthermore, most GCTs involved the index finger and the thumb was spared. This is in contrast with the findings regarding the thumb and index fingers as the main location of GCTs in our study. This laterality of GCTs needs to be explored further with a sample number bigger than the 21 cases reported in our study.
Lipomas are clinically important tumors as they can mimic a soft tissue sarcoma, which if missed can be disastrous for the patient (see figure 2A, B). Any part of the body where adipose tissue is present can be involved (3). Lipomas usually grow slowly and are often of a large size at presentation; however, if the growth is rapid, leading to a large size with pain, this should raise suspicion of a malignant lesion $(4,14)$. Such lesions can cause compression of vital structures such as the median nerve leading to carpal tunnel syndrome, or the ulnar nerve in the Guyon canal resulting in the development of lower ulnar nerve palsy $(1,3,4)$. The mean age for our patients with lipomas was slightly higher than the mean age for all the patients with hand masses. Cavit et al. (1) reported a similar mean age of 51.3 years. This late presentation might be due to the painless nature and slow growth of lipomas. There was no relationship between sex and diagnosis or between side and diagnosis.

The tumor-like conditions found were synovitis resulting from tuberculosis and inflammatory conditions. These are often not considered as tumors, however, patients with tuberculous synovitis can present with localized or diffuse swelling on the dorsum of the wrist. Coulibaly et al. (15) reported on four cases of tuberculous tenosynovitis of the wrist and hand, where one of their patients had a tuberculous mass on the palmar aspect of the hand with signs of median nerve compression. We also found four cases of tuberculous tenosynovitis of the hand and this diagnosis with diffuse swelling of the wrist must be considered, especially with a background of immunosuppression. The differential diagnosis includes many inflammatory conditions but can also mimic an EWS (16). The isolation of the organism from synovial fluid or synovial biopsy forms the basis of the definitive diagnosis (16).

Notwithstanding the fact that the glomus tumor comprises only $1.18 \%(2 / 169)$ of our cases, it has a very significant impact on the affected digit. It is a painful benign tumor from the modified smooth muscles of the glomus body with a slow growth (17). The usual clinical presentation is a solitary nodule in the nail bed that is extremely painful on touching or exposure to cold in the 
Review Of Histology Results Of Hand Masses

fingertip. Treatment is by meticulous surgical excision aberrant germs of cartilage, and are composed of wellwith partial removal of the nail to avoid recurrence (18). differentiated hyaline cartilage (19-21).

With regard to bone masses, most were enchondromas, Enchondromas are usually solitary masses often found as in reported studies where the majority were in the proximal phalanx followed by the metacarpals, enchondromas representing from $30 \%$ to $60 \%$ of all but can present as multiple lesions as in Ollier's disease bone tumors of the hand $(1,19)$. These benign masses and Maffucci. are intramedullary hamartomas, originating from
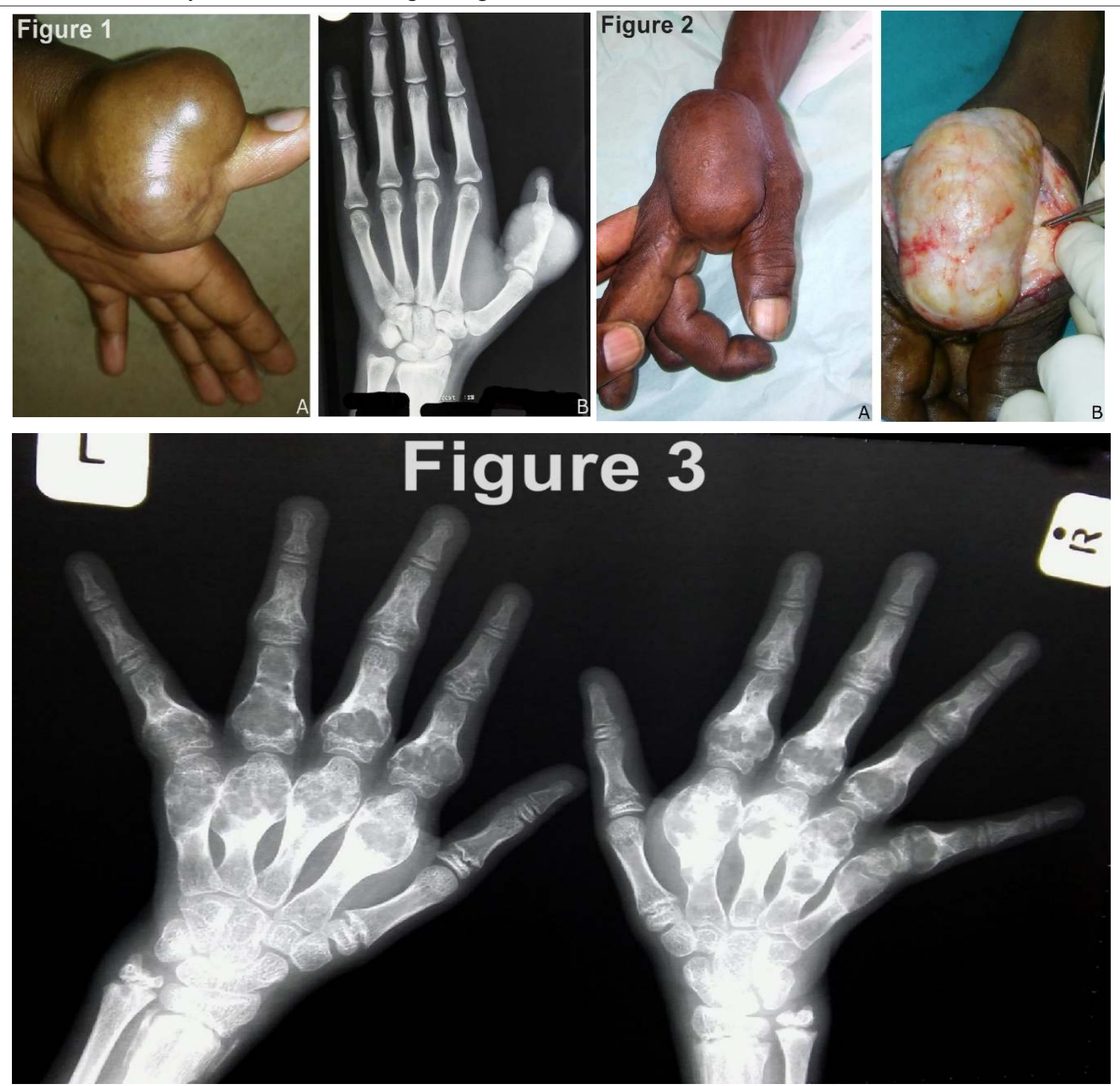

Figure $1 \mathrm{~A}, \mathrm{~B}$. A giant cell tumor of the left thumb (A) with erosion of the proximal phalanx on an anteroposterior X-ray view; Figure 2 A, B. A lipoma of the right first webspace (A) and an intraoperative view (B); Figure 3. Anteroposterior radiological view of the right and left hands showing multiple enchondromas in a case of Ollier disease 
syndrome (20). One of our cases had Ollier's disease (Figure 3). Most patients present with swelling and pain, or a pathological fracture of the digit involved (20). Definitive treatment is surgical curettage combined with a variety of ways to deal with the resultant cavity (19). Options include leaving the cavity to heal by secondary intention or using bone grafts to fill the cavity $(19,21)$. Chondrosarcomas are the most common malignant bone tumors occurring in the hand and can result from malignant transformation of enchondromas in patients with Maffucci syndrome (2, 20, 21). Very few cases of malignant bone lesions of the hand are mentioned in the literature, and our study found only one case of EWS. EWS of the hand is relatively rare and presents with pain, swelling, a mass, or with clinical features of infection with fever and elevated inflammatory markers (22). These signs could be confused with a case of tuberculous tenosynovitis in regions where tuberculosis is endemic (22). Proper biopsy-guided management is of the utmost importance. Definitive treatment is generally a combination of surgery with ray amputation of a digit and adjuvant chemotherapy.

The weakness of this study is in its retrospective nature, with a small sample size. Patient selection eliminated cases diagnosed clinically and managed in the outpatient clinic.

\section{Conclusion}

This study shows that the profile of patients with hand masses from a high-volume hand unit in Johannesburg is similar to what is reported in the literature. Most hand masses in this study were benign soft tissue tumors and GC was the most common type of hand mass, with the majority of patients presenting during the third to fifth decade of life. While no association was found between the diagnosis and sex, or side and diagnosis, it was notable that in the case of GCT there was a significant difference in distribution in favor of the right side of the hand, a finding which needs to be further explored.

Footnote: This work forms part a research report submitted to the university of the Witwatersrand for the degree of Master of Medicine in Plastic and Reconstructive Surgery.

\section{References}

1. Cavit A, Özcanli H, Sançmiş $M$, et al. Tumorous conditions of the hand: A retrospective review of 402 cases. Turk Patoloji Derg. 2018; 34(1): 66-72.

2. Puhaindran ME, Athanasian EA. Malignant and metastatic tumors of the hand. J Hand Surg Am. 2010; 35(11): 1895-1900.

3. Chatterton BD, Moores TS, Datta P, et al. An exceptionally large giant lipoma of the hand. BMJ Case Rep. 2013; 2013: bcr2013200206.

4. Sbai MA, Benzarti S, Msek H, et al. Carpal tunnel syndrome caused by lipoma: a case report. Pan Afr Med J. 2015; 22: 51.

5. Meena S, Gupta A. Dorsal wrist ganglion: Current review of literature. J Clin Orthop Trauma. 2014; 5(2): 59-64.

6. Lee $\mathrm{CH}$, Tandon A. Focal hand lesions: review and radiological approach. Insights Imaging. 2014; 5(3): 301319.

7. Fletcher CDM, Bridge JA, Hogendoorn PCW et al. WHO Classification of Tumours of Soft Tissue and Bone: WHO Classification of Tumours, 4th Edition, 2013; Vol. 5. Geneva: WHO

8. Payne WT, Merrell G. Benign bony and soft tissue tumors of the hand. J Hand Surg Am. 2010; 35(11): 1901-1910.

9. Kuliński S, Gutkowska O, Mizia S, et al. Ganglions of the hand and wrist: Retrospective statistical analysis of 520 cases. Adv Clin Exp Med. 2017; 26(1): 95-100.

10. Al-Qattan MM. Giant cell tumours of tendon sheath: classification and recurrence rate. J Hand Surg Am. 2001; 26(1): 72-75.

11. Suresh SS, Zaki H. Giant cell tumor of tendon sheath: case series and review of literature. J Hand Microsurg. 2010; 2(2): 67-71.

12. Darwish FM, Haddad WH. Giant cell tumour of tendon sheath: experience with 52 cases. Singapore Med J. 2008; 49(11): 879-882.

13. Tang $\mathrm{ZH}$, Rajaratnam V, Desai V. Incidence and anatomical distribution of hand tumours: a Singapore study. Singapore Med J. 2017; 58(12): 714-716.

14. Hsu CS, Hentz VR, Yao J. Tumours of the hand. Lancet Oncol. 2007; 8(2): 157-166.

15. Coulibaly NF, Ba A, Gueye AB, et al. Tuberculous tenosynovitis of the wrist and the hand: The 3 anatomoclinical forms described by Kanavel (about 4 cases). J Orthop Case Rep. 2017; 7(6): 68-72.

16. Sanders CJ, Schucany WG. Tuberculous tenosynovitis. Proc Bayl Univ Med Cent. 2008; 21(1): 71-72.

17. White CP, Jewer DD. Atypical presentation of a glomus tumour: a case report. Can J Plast Surg. 2006; 14(4): $237-$ 238.

18. Fujioka H, Kokubu T, Akisue $\mathrm{T}$, et al. Treatment of subungual glomus tumor. Kobe J Med Sci. 2009; 55(1): E1-E4. 
19. Nagura I, Kanatani T, Sumi M, et al. Recurrence of enchondroma in a middle finger after curettage and backfilling with calcium phosphate bone cement: A case report. Kobe J Med Sci. 2016; 62(1): E19-E21.

20. Sassoon AA, Fitz-Gibbon PD, Harmsen WS, et al. Enchondromas of the hand: factors affecting recurrence, healing, motion, and malignant transformation. J Hand Surg Am. 2012; 37(6): 1229-1234.

21. Sağlık Y, Atalar H, Armangil M, et al. Management of tumors and tumor-like lesions of the hand: a review of 191 patients. Eklem Hastalik Cerrahisi. 2013; 24(3): 149-155.

22. Rajappa S, Menon P, Sundaram S. Ewings sarcoma of the hand - A case report. J Hand Microsurg. 2010; 2(2) :8284. 